\title{
Multi-period supplier selection under price uncertainty
}

\author{
Alper Şen ${ }^{1 *}$, Hande Yaman $^{1}$, Kemal Güler ${ }^{2}$ and Evren Körpeoğlu ${ }^{2}$ \\ ${ }^{1}$ Bilkent University, Ankara, Turkey; and ${ }^{2}$ Hewlett-Packard Laboratories, Palo Alto, USA
}

\begin{abstract}
We consider a problem faced by a procurement manager who needs to purchase a large volume of multiple items over multiple periods from multiple suppliers that provide base prices and discounts. Discounts are contingent on meeting various conditions on total volume or spend, and some are tied to future realizations of random events that can be mutually verified. We formulate a scenario-based multi-stage stochastic optimization model that allows us to consider random events such as a drop in price because of the most favoured customer clauses, a price change in the spot market or a new discount offer. We propose certainty-equivalent heuristics and evaluate the regret of using them. We use our model for three bidding events of a large manufacturing company. The results show that considering most favored customer clauses in supplier offers may create substantial savings that may surpass the savings from regular discount offers.
\end{abstract}

Journal of the Operational Research Society (2014) 65(11), 1636-1648. doi:10.1057/jors.2013.111

Published online 25 September 2013

Keywords: supplier selection; quantity discounts; price uncertainty; most favoured customer; multi-stage stochastic programming

\section{Introduction}

Procurement is a core strategic function that affects the profitability of a firm, as the cost of goods and services acquired through procurement typically constitutes a majority of operating expenses. A fundamental problem in procurement is supplier selection or sourcing, that is, how to allocate the firm's business across suppliers, considering factors such as cost, quality, responsiveness and risk.

The industry has seen two shifts in supplier selection during the past two decades. First, with the advent of the Internet, procurement organizations moved away from manual bidding processes and negotiations to electronic sourcing. For example, between 2001 and 2006, Hewlett-Packard increased its total spend through e-sourcing events to US\$30 billion, a 100-fold increase (Carbone, 2004; Moody, 2006). Electronic sourcing not only allowed firms to expand their supply pool to more competition, but also made the supplier selection process faster and more structured, enabling a simultaneous evaluation and negotiation of supplier offers. A second shift is an organizational change with which corporations started to procure centrally to leverage economies of scale of their global business. For example, Hewlett-Packard combined even its indirect and services procurement globally and started to use category-based sourcing to leverage its total spend of $\$ 16.5$ billion (Avery, 2008). While these two shifts increased the

\footnotetext{
*Correspondence: Alper Şen, Department of Industrial Engineering, Bilkent University Bilkent, Ankara, 06800, Turkey.

E-mail: alpersen@bilkent.edu.tr
}

potential value of the procurement function, they also made the decisions faced by procurement managers more complex and challenging. A procurement manager now needs to distribute a large volume of many items required at multiple locations across many global suppliers that approach the firm with various terms and offers.

A complicating feature of this problem is regarding how the suppliers present their price offers in a procurement environment. Many suppliers often exhibit economies of scale and scope in their production activities. Some others have growth and market share targets for a specific group of items. Suppliers express these internal efficiencies and pressures by offering discounts to the buyer. These offers are usually complex and are contingent on meeting various conditions on total available market, volume or spend for a single item or a set of items, and the discounts may be applicable to the same or a different set of items.

It is not possible to incorporate these complex discount offers into a simple reverse auction. Ignoring these (potential) offers and selecting the lowest bidding supplier for each item or lot would lead to inefficiencies. We have recently seen efforts to develop tools that would enable suppliers to express these offers and buyers to evaluate them. The success of the software tool CombineNet is described in Sandholm (2007). Between 2001 and 2006, 447 bidding events are administered totalling a spend of $\$ 35$ billion. It is believed that CombineNet delivered savings of $\$ 4.4$ billion in these events. Bichler et al (2011) proposed a bidding language and an optimization model to express and evaluate more complex offers. 
Both of these efforts and many other research in this area assume that the important parameters of the problem are perfectly known in advance. However, procurement environments are replete with uncertainties. A primary uncertainty is in the volume that needs to be procured. Since the demand for end-products is often volatile, it is also very hard to predict the amounts of goods and services that need to be procured to make them. For example, global shipments of personal computers declined in the first quarter of 2011, by 3.2\% according to an estimate by International Data Corp. and by $1.1 \%$ according to an another estimate by Gartner Inc., while both tracking firms previously predicted an increase (Sherr, 2011).

Another important uncertainty is in the prices of components that need to be procured. For example, prices of many components that are used in personal computers fluctuate heavily and these shifts in prices are also very hard to predict. The price of the DRAM memory that Hewlett-Packard uses dropped by over $90 \%$ in 2001, and then more than tripled in early 2002 (Nagali et al, 2008). Supplier offers usually state a commitment to base prices and discounts that will be given if certain conditions are met. However, price uncertainty in the market may still have an impact on how a procurement manager would evaluate such offers through a number of ways. First, if the procurement is through a contracted supplier, the future prices of this supplier to other customers may have an impact on an existing contract based on certain clauses. These clauses usually refer to what is known as a most-favored-customer (MFC) status. A customer who obtains a MFC status from a company is guaranteed to receive the best price the company gives to anyone. If the supplier lowers price to someone else, then the customer's price will be lowered to match. In some cases, a customer may need to purchase a minimum volume of a set of items over a specified time period to obtain this status. In other cases, the customer (such as a government agency) may demand MFC status for any contract without any condition. Lowest prices can be verified and contract compliance can be ensured through third-party audits.

MFC clauses are commonly used in procurement contracts in many industries. For example, a contract (Sample Business Contracts, 2012) between Cisco Systems Inc. and one of its suppliers, Frontier Software Development Inc., stipulates 'Frontier represents and warrants to Cisco that the product prices/ license fees offered to Cisco under this agreement are no less favorable than the product prices/license fees offered to any other party purchasing or licensing similar quantities. In the event, Frontier offers more favorable product prices/license fees to any other party. Frontier will promptly notify Cisco of such event and offer such more favorable product prices/license fees to Cisco. Commencing upon the date, such more favorable product prices/license fees were offered to the other party'.

As an example of a contract that guarantees a government agency to purchase at the lowest price, we note the following price reductions clause stated in a contract between Hewlett-Packard and US Department of Defense (DoD-ESI, 2012): 'The prices under this BPA (blanket purchase agreement) shall be at least as low as the prices that the contractor has under any other contract instrument under like terms and conditions. If at any time the prices under any other contract instrument become lower than the prices in this BPA, this BPA will be modified to include the lower prices'. Governments usually enforce the compliance to these clauses strictly. In a recent settlement, Oracle accepted to pay the US government $\$ 199.5$ million after a file suit claiming that the company was not providing the government discounts that were as deep as some other customers were receiving (Montalbano, 2011).

Another contract clause that price uncertainty can affect is what is called meet-the-competition-clause (MCC). An MCC clause (sometimes also referred to as meet-or-release clause) in a procurement contract gives the seller an option to retain the customer's business by matching any lower price offer that may be coming in the future. A third contract type that may lead to uncertainty in price is price indexing. In this case, the contract price of a product is indexed to the price of a commodity or an official price index (such as consumer price index). For example, in the United Kingdom, 85\% of natural gas is sold under long-term contracts in which prices are indexed to the spot market (Neumann and von Hirschhausen, 2004). Uncertainty in the commodity prices or price index obviously creates uncertainty on the prices that the seller would charge to the buyer. Finally, if some of the products under consideration are commodity-like products and can also be procured from the spot market, an uncertainty in spot prices has a clear and direct effect on how much the manager should procure from the spot market or using a fixed-price contract now and in the future.

In this study, we develop a model that incorporates price uncertainty to the supplier selection problem when the suppliers offer complex discount offers. The formal problem we consider may be stated as follows. A buyer needs to purchase a large volume of multiple items over a planning horizon (a quarter or a year) that consists of multiple periods (months). The demand for each item can be different in each period, but is known. There are multiple suppliers that are qualified to offer all or a portion of the items in consideration. Each supplier provides a base price for each offered item. In addition, suppliers propose various discount offers to the buyer that are contingent on meeting various conditions on a single item or a group of items, over a single period or multiple periods. An offer may provide per unit discounts that can be applied to a single item or a group of items, over a single period or multiple periods, and on all units purchased (all-units discount) or units purchased above a threshold (incremental discounts). Alternatively, an offer may provide the buyer a lump sum. Some of these offers may be tied to realization of random events in the future that can be mutually verified. The buyer's problem is to select suppliers and determine the amount of each item to be procured from each supplier in each period of the planning horizon to minimize its expected procurement and inventory holding costs while satisfying item demands in each period. There could also be capacity constraints that limit how much the buyer can 
procure from a supplier. In addition, the buyer may also enforce certain side constraints (eg, enforce a minimum and maximum number of suppliers for each item) to properly manage other procurement risks. While the procurement decisions for the first period are executed immediately, the decisions in the latter periods will be contingent on the realization of random events in those periods (ie, recourses).

We formulate the buyer's problem as a multi-stage stochastic mixed-integer programme using a scenario tree. To the best of our knowledge, this is the first model for the supplier selection problem that simultaneously considers uncertainty and discount offers of combinatorial nature. This is also one of the first multi-period models and allows the buyer to consider discount rules defined over multiple periods and carry inventory from one period to another to be eligible for a favourable discount. The formulation is also very general in two aspects. First, we can represent a variety of random events that have direct or indirect effects on the discounts that the buyer gets from the suppliers. Second, we can represent many different forms of supplier offers with very complex conditions and discounts. For example, the model supports the separation of items (periods) for which the conditions are imposed and items (periods) on which the discounts apply, pricing with multiple price breaks and incremental or all-units discounts.

We also suggest two certainty-equivalent heuristics that can be used for this problem. In both of these heuristics, a deterministic version of the problem is solved by setting the prices in later periods to their expected values. The static heuristic solves the problem only once at the beginning of the horizon and does not respond to the actual realizations of events in the later periods. The dynamic heuristic, on the other hand, resolves the problem at each period. We show how to compute the performance of these heuristics by using our stochastic formulation.

Our work is motivated by our experience with a major manufacturing company that conducts quarterly bidding events to select suppliers for its global demand for various components. In all of these procurement events, suppliers' bids include various volume discounts in addition to base prices for each component. Some of these discount offers are very complex in nature and are contingent on meeting multiple conditions on multiple items. Furthermore, some of the suppliers offer contracts with MFC terms that guarantee the lowest price provided that the manufacturing company procures enough volume from them. We used our multi-stage stochastic programming model to evaluate MFC status benefits and regular discount offers for three bidding events that took place in 2010. The results of this preliminary study show that considering MFC terms in addition to the regular discount offers may lead to substantial savings. In some of the events, the incremental savings by taking MFC terms into account may be larger than the savings that can be obtained by only evaluating regular discount offers. The results also show that the price certainty-equivalent heuristics, the static version in particular, fail to capture the benefits of MFC terms in contracts.
The rest of the paper is organized as follows. In Section 2, we analyse a single-item, two-period problem with two suppliers to gain insight into the trade-offs. In Section 3, we review the literature on supplier selection problem. In Section 4, we present our model. In Section 5, we propose the two certainty-equivalent heuristics. In Section 6, we discuss the implementation of our model at a major manufacturing company and analyse the effects of various model elements and parameters on the benefits of considering MFC terms. We conclude in Section 7.

\section{A motivating example}

In order to explain the basic trade-offs in evaluating discount offers under uncertainty, and to show the need to use a formal stochastic model to support decision making in this context, we provide the following stylized example.

A company needs to procure an item over a two-period horizon. The demands in the first and second periods are $\delta_{1}$ and $\delta_{2}$, respectively. There are two suppliers that offer this item. Supplier $a$ charges $\mu_{a}$ per unit and offers a most favoured customer clause in the contract. Under this contract, if the firm procures $100 \mathrm{~m}$ percent of its demand from supplier $a$ in Period 1, it will benefit from any possible reduction in price (to other customers) in Period 2. The price per unit will be reduced by $\pi_{a}$ with a probability $\gamma$ and will remain constant with probability $1-\gamma$. Supplier $b$ charges $\mu_{b}$ per unit and offers a volume discount contract. Under this contract, if the firm procures a total of $\rho$ in two periods from supplier $b$, it will receive a discount of $\pi_{b}$ per unit. We assume that

$$
\delta_{1}+\delta_{2} \geqslant \rho>(1-m) \delta_{1}+\delta_{2} .
$$

That is, (i) the firm can always qualify for a volume discount from supplier $b$ by buying enough; and (ii) the firm cannot qualify for the volume discount offer from supplier $b$ and buy enough from supplier $a$ to benefit from a possible price drop at the same time. We also assume that

$$
\mu_{b}<\mu_{a}<\mu_{b}-\pi_{b}+\pi_{a},
$$

that is, firm will choose supplier $a$ over $b$ if MFC clause in supplier $a$ is used, and supplier $b$ over supplier $a$, otherwise. If the firm buys enough from supplier $a$ to benefit from a potential price drop, its expected cost will be

$$
\Phi_{0}^{a}=\mu_{a} m \delta_{1}+\mu_{b}(1-m) \delta_{1}+\gamma\left(\mu_{a}-\pi_{a}\right) \delta_{2}+(1-\gamma) \mu_{b} \delta_{2} .
$$

On the other hand, if the firm chooses to use supplier $b$ and benefit from the volume discount offer, its cost will be

$$
\Phi^{b}=\left(\mu_{b}-\pi_{b}\right)\left(\delta_{1}+\delta_{2}\right) .
$$

The firm will opt for supplier $a$ 's MFC clause $\left(\Phi_{0}^{a}<\Phi^{b}\right)$ if and only if

$$
\gamma>\gamma_{0}=\frac{\pi_{b}\left(\delta_{1}+\delta_{2}\right)+\left(\mu_{a}-\mu_{b}\right) m \delta_{1}}{\left(\pi_{a}+\mu_{b}-\mu_{a}\right) \delta_{2}} .
$$


An alternative to using the stochastic formulation is to use a certainty-equivalent argument and assume that supplier $a$ 's second period price will be the expected price $\mu_{a}-\gamma \pi_{a}$. In this case, we can write firm's cost if it chooses to use the MFC clause as

$$
\Phi_{1}^{a}=\mu_{a} m \delta_{1}+\mu_{b}(1-m) \delta_{1}+\left(\mu_{a}-\gamma \pi_{a}\right) \delta_{2} .
$$

Hence, the firm will opt for supplier $a$ 's MFC clause $\left(\Phi_{1}^{a}<\Phi^{b}\right)$ if and only if

$$
\gamma>\gamma_{1}=\frac{\pi_{b}\left(\delta_{1}+\delta_{2}\right)+\left(\mu_{a}-\mu_{b}\right) m \delta_{1}+\left(\mu_{a}-\mu_{b}\right) \delta_{2}}{\pi_{a} \delta_{2}} .
$$

Denoting $\alpha=\pi_{b}\left(\delta_{1}+\delta_{2}\right)+\left(\mu_{a}-\mu_{b}\right) m \delta_{1}, \beta=\left(\pi_{a}+\mu_{a}-\mu_{b}\right) \delta_{2}$ and $\lambda=\left(\mu_{a}-\mu_{b}\right) \delta_{2}$, we have $\gamma_{0}=\alpha / \beta$ and $\gamma_{1}=(\alpha+\lambda) /(\beta+\lambda)$. It is then easy to see that $\gamma_{0}$ is strictly smaller than $\gamma_{1}$ if and only if $\gamma_{0}<1$, that is, unless it is never optimal for the firm to consider the supplier $a$, which offers the MFC clause. In this case for $\gamma_{0}<\gamma \leqslant \gamma_{1}$, the firm's optimal action is to procure $m \delta_{1}$ from supplier $a$ in Period 1. However, this action will not be taken if a certainty-equivalent approach is used. In general, the decisions taken using a certainty-equivalent approach would be different from optimal decisions (using stochastic formulations) and therefore lead to expected costs that are higher than optimal.

\section{Literature review}

The impact of quantity discounts on replenishment and procurement decisions of a company is well studied in the operations management literature (Munson and Rosenblatt, 1998). Most of the basic textbooks in this area include a section on extensions of the economic order quantity (EOQ) model that consider quantity discounts (eg, Silver et al, 1998, §5.5). Another line of research focuses on sourcing, that is, how a company should select and allocate its spend to different suppliers based on different factors such as cost, quality, lead time and reliability (Chopra and Meindl, 2013, §13).

While the extensions of the EOQ model for the case of quantity discounts and single-item, single-supplier problems are usually tractable, the problem becomes difficult, even for the case of a single item when there are multiple suppliers whose prices are functions of the quantity purchased. For example, consider a buyer who needs to purchase a predetermined amount of a single item from a set of suppliers. Each supplier offers a certain price, but the price is valid only if the quantity purchased is in a specific interval, reflecting the cost and capacity structure of the supplier. Chauhan et al (2005) show that the problem is NP-hard.

For reviews on the supplier selection problem, we refer the reader to Benton and Park (1996), Munson and Rosenblatt (1998) and Aissaoui et al (2007). Here we summarize some examples on different variants of the problem.

Most studies on supplier selection for multiple items consider single-period problems. Goossens et al (2007) study the problem of deciding on purchase quantities for multiple items from multiple suppliers that offer total quantity discounts based on total purchase quantities. The authors prove that this problem is NP-hard even for some specific discount structures. They present a Mixed-Integer Linear Programming (MILP) model and a branch-and-bound procedure based on a minimum cost network flow problem reformulation of the continuous relaxation. They extend their results to variants of the problem with market share constraints, limited number of winning suppliers and multiple periods. Manerba and Mansini (2012) study the same problem under capacity constraints and present new valid inequalities. They propose a branch-and-cut algorithm and a hybrid heuristic to provide an initial feasible solution to the exact approach. Qin et al (2012) study a distribution planning problem where shipping companies offer total quantity discounts. Crama et al (2004) consider the supplier selection problem with alternative product recipes and Mansini et al (2012) incorporate transportation costs. A different setting where suppliers offer their products in bundles is studied by Murthy et al (2004). In this problem, decisions regarding the purchase quantities for different items are related not only through bundles but also through fixed costs of buying from suppliers. A Lagrangian relaxation-based heuristic is proposed to solve this problem. Sadrian and Yoon (1994) and Katz et al (1994) present MILP formulations for the problem in the presence of business volume discounts. Bichler et al (2011) introduce a comprehensive bidding language that allows for elaborate discount structures. Total quantity and incremental quantity discounts, as well as lump sum discounts and markups, with conditions on spend or purchase quantities can be expressed with this bidding language. The authors present a MILP model to solve the supplier selection problem and report the results of their experiments in solving the model under different scenarios.

There are few studies on the supplier selection problem with multiple periods and dynamic demand. Tempelmeier (2002) presents formulations and a heuristic solution approach for the single-item problem with both total quantity discounts and incremental discounts. Moreover, van de Klundert et al (2005) study the problem of selecting telecommunications carriers under total quantity discounts. The discounts are given based on the total call minutes over the planning horizon; however, lower and upper bounds are imposed on call minutes per period routed via each carrier.

The multi-item problem with dynamic demand is studied by Stadtler (2007). Different from our study, discount rules involve a single item, and decisions concerning different items depend on a fixed cost of buying from suppliers. Xu et al (2000) study the single supplier problem in the presence of business volume discounts and setup costs. Rong et al (2012) study an interesting multi-item problem where the discounts are due to ordering in standard batches (eg, full pallets) and the buyer needs to determine the procurement strategy for each item to minimize procurement, transportation, inventory and material handling costs.

Most research on supplier selection problems with discount offers assume a deterministic setting. When the demand is 
stochastic, existence of a contract with volume discounts entangles the inventory decisions of a buyer. For example, see Bassok and Anupindi (1997) who analyse a supply contract of a single product in which the cumulative orders over multiple periods should be larger than a prespecified quantity in order to qualify for a discount. The authors extend their work (Anupindi and Bassok, 1998) to the case of multiple products with business volume discounts.

The present study extends the literature on multi-item problems with multiple suppliers and dynamic demand by considering very general discount rules and price uncertainty.

\section{The multi-stage stochastic programming model}

A firm (buyer) needs to procure a set of items $\mathcal{I}$ over a set of periods $\mathcal{T}$. For each item $i \in \mathcal{I}$ and period $t \in \mathcal{T}$, the firm has to satisfy a demand denoted by $\delta_{i t}$ (a deterministic quantity) without a backlog. The firm works with a set of suppliers $\mathcal{N}$. For each item $i$, there is a subset of suppliers $\mathcal{N}_{i} \subseteq \mathcal{N}$ that are qualified. Supplier $j$ in $\mathcal{N}_{i}$ charges a unit price $\mu_{i j t}$ and has a capacity $\kappa_{i j t}$ for item $i$ in period $t$. The firm can also carry inventory from period $t$ to period $t+1$ incurring an inventory holding cost of $\eta_{i t}$ for each unit of item $i$ 's ending inventory in period $t$.

A set of discount rules $\mathcal{D}$ and a set of lump sum rebate rules $\mathcal{L}$ are available. These rules involve a set of conditions $\mathcal{C}$ on order quantities. The quantity $\rho_{c}$ is the minimum order quantity that the firm needs to purchase from items in set $\mathcal{I}_{c} \subseteq \mathcal{I}$ over periods $\mathcal{T}_{c} \subseteq \mathcal{T}$ for condition $c \in \mathcal{C}$ to be satisfied. Let $\varepsilon$ be the set of conflicting pairs of rules.

We define $\mathcal{R}=\mathcal{D} \cup \mathcal{L}$. The buyer can benefit from the rule $r$ offered by supplier $j(r) \in \mathcal{N}$ if it satisfies the conditions $\mathcal{C}_{r} \subseteq \mathcal{C}$ and if it does not benefit from any other rule in $\mathcal{R}_{r} \subset \mathcal{R}$. If $r \in \mathcal{L}$, then a lump sum rebate of $\omega_{r}$ is offered. If $r \in \mathcal{D}$, the supplier provides a set of discounts $\mathcal{K}_{r}$. The discount $k \in \mathcal{K}_{r}$ reduces the price by $\pi_{k}$ per unit for items in the set $\mathcal{I}_{k} \subseteq \mathcal{I}$ purchased over periods $\mathcal{T}_{k} \subseteq \mathcal{T}$ exceeding the quantity $\theta_{k}$ $\left(\mathcal{I}_{k 1} \cap \mathcal{I}_{k 2}=\emptyset\right.$ for all $\left.k_{1}, k_{2} \in \mathcal{K}_{r}, k_{1} \neq k_{2}\right)$.

Let $\mathcal{V}$ be the set of nodes of the scenario tree with node 0 corresponding to the root and $\mathcal{V}_{t}$ be the set of nodes in layer $t \in \mathcal{T}$. For a given node $s \in \mathcal{V}$, let $\tau(s)$ be its layer, $a(s)$ be its predecessor in the scenario tree, $\mathcal{P}^{s}$ be the set of nodes on the path from the root to node $s$ and $\gamma_{s}$ be its probability. We define the rules at the terminal nodes. Let $\mathcal{R}^{s}$ be the set of discount rules available at node $s$. Define $\mathcal{D}^{s}=\mathcal{D} \cap \mathcal{R}^{s}$ and $\mathcal{L}^{s}=\mathcal{L} \cap \mathcal{R}^{s}$. Let $\mathcal{E}^{s}$ be the set of pairs of conflicting rules at node $s$, that is, $\mathcal{E}^{s}=\mathcal{E} \cap\left(\mathcal{R}^{s} \times \mathcal{R}^{s}\right)$.

We show the construction of the scenario tree for the example problem discussed in Section 1 in Figure 1. Here, $\mathcal{N}=\{a, b\}, \mathcal{T}=\{1,2\}, \mathcal{I}=\{1\}, \quad \mathcal{V}=\{0,1,2\}$, $a(1)=a(2)=0, \tau(0)=1, \tau(1)=\tau(2)=2$. Supplier $a$ offers the discount rule $r_{1}$. The discount rule $r_{1}$ is available only at node 2 (if supplier $a$ lowers its price to other customers) contingent on conditions in set $\mathcal{C}_{r_{1}}=\left\{c_{1}\right\}$. Condition $c_{1}$ states that the

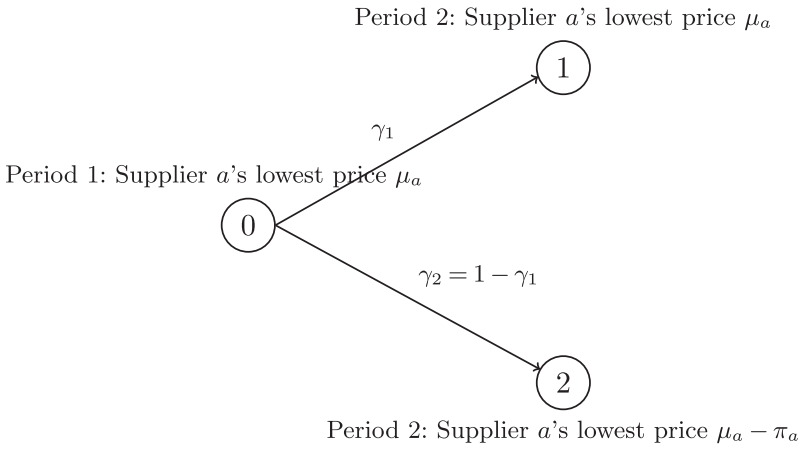

Figure 1 Scenario tree for the example problem of Section 2.

volume of products $\mathcal{I}_{c_{1}}=\{1\}$ in periods $\mathcal{T}_{c_{1}}=\{1\}$ purchased from supplier $a$ exceeds $\rho_{c_{1}}=m \delta_{1}$. Rule $r_{1}$ allows discounts in the set $\mathcal{K}_{r_{1}}=\left\{k_{1}\right\}$. Discount $k_{1}$ provides a discount of $\pi_{k_{1}}=\pi_{a}$ per unit for products $\mathcal{I}_{k_{1}}=\{1\}$ bought in quantity above $\theta_{k_{1}}=0$ in periods $\mathcal{T}_{k_{1}}=\{2\}$. Supplier $b$ offers the discount rule $r_{2}$. The discount rule $r_{2}$ is available in nodes 1 and 2 and is contingent on conditions in set $\mathcal{C}_{r_{2}}=\left\{c_{2}\right\}$. Condition $c_{2}$ states that the volume of products $\mathcal{I}_{c_{2}}=\{1\}$ in periods $\mathcal{T}_{c_{2}}=\{1,2\}$ purchased from supplier $b$ exceeds $\rho_{c_{2}}=\rho$. Rule $r_{2}$ allows discounts in the set $\mathcal{K}_{r_{2}}=\left\{k_{2}\right\}$. Discount $k_{2}$ provides a discount of $\pi_{k_{2}}=\pi_{b}$ per unit for products $\mathcal{I}_{k_{2}}=\{1\}$ bought in quantity above $\theta_{k_{2}}=0$ in periods $\mathcal{T}_{k_{2}}=\{1,2\}$.

At the beginning of horizon, the orders are placed for the first period. Recourse actions are taken at the beginning of each other period based on actual realizations. We define the following decision variables. The quantity $x_{i j s}$ is the order quantity for item $i \in \mathcal{I}$ and supplier $j \in \mathcal{N}_{i}$ at node $s \in \mathcal{V}$. $I_{\text {is }}$ stands for the ending inventory for item $i \in \mathcal{I}$ at node $s \in \mathcal{V}$. The binary variable $z_{r}^{s}$ takes value 1 if rule $r \in \mathcal{R}^{s}$ applies at node $s \in \mathcal{V}$ and takes value 0 otherwise. Finally, $y_{k}^{s}$ is the total amount of units of items in the set $\mathcal{I}^{k}$ that are discounted with discount $k \in \mathcal{K}_{r}$ as a part of rule $r \in \mathcal{D}^{s}$ at node $s \in \mathcal{V}$, that is, $y_{k}^{s}=\left(\sum_{i \in \mathcal{I}_{k}} \sum_{\hat{s} \in \mathcal{P}^{s}: \tau(\hat{s}) \in \mathcal{T}_{k}} x_{i j(r) \hat{s}}-\theta_{k}\right)^{+} z_{r}^{s}$. Now we can model our problem as follows:

$$
\begin{gathered}
\min \sum_{s \in \mathcal{V}} \gamma_{s}\left(\sum_{i \in \mathcal{I}} \sum_{j \in \mathcal{N}_{i}} \mu_{i j \tau(s)} x_{i j s}+\sum_{i \in \mathcal{I}} \eta_{i \tau(s)} I_{i s}-\sum_{r \in \mathcal{D}^{s}} \sum_{k \in \mathcal{K}_{r}} \pi_{k} y_{k}^{s}-\sum_{r \in \mathcal{L}^{s}} \omega_{r} z_{r}^{s}\right) \\
\text { s.t. } I_{i s}=I_{i a(s)}+\sum_{j \in \mathcal{N}_{i}} x_{i j s}-\delta_{i \tau(s)} \quad \forall i \in \mathcal{I}, s \in \mathcal{V}, \\
x_{i j s} \leqslant \kappa_{i j \tau(s)} \quad \forall i \in \mathcal{I}, j \in \mathcal{N}_{i}, s \in \mathcal{V},
\end{gathered}
$$

$$
\begin{aligned}
& \sum_{i \in \mathcal{I}_{c}} \sum_{\hat{s} \in \mathcal{P}^{s}: \tau(\hat{s}) \in \mathcal{T}_{c}} x_{i j(r) \hat{s}} \geqslant \rho_{c} z_{r}^{s} \quad \forall s \in \mathcal{V}, r \in \mathcal{R}^{s}, c \in \mathcal{C}_{r}, \\
& y_{k}^{s} \leqslant \sum_{i \in \mathcal{I}_{k}} \sum_{\hat{s} \in \mathcal{P}^{s}: \tau(\hat{s}) \in \mathcal{T}_{k}} x_{i j(r) \hat{s}}-\theta_{k} z_{r}^{s} \\
& \forall s \in \mathcal{V}, r \in \mathcal{D}^{s}, k \in \mathcal{K}_{r},
\end{aligned}
$$




$$
\begin{gathered}
y_{k}^{s} \leqslant\left(\sum_{i \in \mathcal{I}_{k}} \sum_{t \in \mathcal{T}_{k}} \kappa_{i j(r) t}-\theta_{k}\right) z_{r}^{s} \quad \forall s \in \mathcal{V}, r \in \mathcal{D}^{s}, k \in \mathcal{K}_{r}, \\
z_{r}^{s}+z_{r^{\prime}}^{s} \leqslant 1 \quad \forall s \in \mathcal{V},\left\{r, r^{\prime}\right\} \in \mathcal{E}^{s} \\
x_{i j s} \geqslant 0 \quad \forall i \in \mathcal{I}, j \in \mathcal{N}_{i}, s \in \mathcal{V} \\
I_{i s} \geqslant 0 \quad \forall i \in \mathcal{I}, s \in \mathcal{V} \\
z_{r}^{s} \in\{0,1\} \quad \forall s \in \mathcal{V}, r \in \mathcal{R}^{s} \\
y_{k}^{s} \geqslant 0 \quad \forall s \in \mathcal{V}, r \in \mathcal{D}^{s}, k \in \mathcal{K}_{r}
\end{gathered}
$$

The objective function (1) is equal to the expected total cost. Constraints (2) are inventory balance equations. The capacities of suppliers are respected due to constraints (3). Constraints (4) impose the minimum order quantity conditions for the rules. Conditions on minimum spend can be modelled similarly. Constraints (5), (6) and (11) compute the amount of discounted units (we assume that $\sum_{i \in \mathcal{I}_{k}} \sum_{\hat{s} \in \mathcal{P}^{s}: \tau(\hat{s}) \in \mathcal{T}_{k}} x_{i j(r) \hat{s}} \geqslant \theta_{k}$ is implied by the conditions for discount rule $r$ and discount $k \in \mathcal{K}_{r}$ ). If, in scenario $s \in S$, discount rule $r \in \mathcal{D}^{s}$ applies, then constraints (6) is redundant and the maximum of 0 and the right hand side of constraint (5) is equal to the amount of discounted units. If this rule does not apply, then constraint (5) is redundant and constraints (6) and (11) force $y_{k r}^{s}$ to 0 . Finally, constraints (7) ensure that conflicting rules do not apply at the same time.

The formulation can be strengthened by replacing conflict constraints (7) with inequalities corresponding to cliques in the conflict graph $\mathcal{G}^{s}=\left(\mathcal{R}^{s}, \mathcal{E}^{s}\right)$. In some cases, the same information can be used to strengthen constraints (4). We sketch this with a very simple example. Suppose that we consider a single period problem where Supplier 1 offers total quantity discounts for Item 1. The unit price reduces by a factor for every 1000 items purchased and the capacity is 4000. To handle this discount, we define four discount rules $r \in\{1, \ldots, 4\}$, each with a single condition. We replace constraint (4) with $x_{110} \geqslant \sum_{r=1}^{4} 1000 r z_{r}^{0}$ and use the clique inequality $\sum_{r=1}^{4} z_{r}^{0} \leqslant 1$.

The MILP formulation given in (1-11) can be used to model various forms of regular discount offers and other discounts that are contingent on the realization of random events. We discuss some of these here. First, traditional quantity discounts schemes can be easily modelled. Consider, for example, an incremental discount rule $r$ that requires condition $c$ and applies a discount $k$. The condition and discount are applied on the same item set and same period set, that is, $\mathcal{I}_{c}=\mathcal{I}_{k}$ and $\mathcal{T}_{c}=\mathcal{T}_{k}$. Thresholds are then also set to be the same, that is, $\rho_{c}=\theta_{k}$. The rule is defined in all terminal nodes. Multiple price breaks can be modelled using multiple rules that are disjoint. An all-units discount rule can be modelled similarly, except that now we set $\theta_{k}=0$. One can also separate the periods (items) for which the conditions are imposed and the periods (items) for which the discounts are applied on.
In addition to MFC terms that are explained by an example in Figure 1, various other uncertainties can be modelled. For the case of index pricing, various scenarios can be created for the value of the index in the later periods. For each scenario, we define a discount rule that provides a discount in the amount of price difference without a condition. One can also model spot price uncertainty by defining a dummy supplier for spot purchases and creating a scenario for each possible price change in the spot market. We can then define a discount rule for every terminal node whose path from the root node has a price change. These rules will also have no conditions and will provide a discount in the amount of price change for all units purchased in periods after the price change took place. Finally, any potential regular discount offer in the future (eg, if the buyer thinks that there is a chance that one of the suppliers will offer a new discount in the middle of the planning horizon) can be easily incorporated in the model provided that the conditions and discounts can be properly estimated.

\section{Heuristics}

A usual approach in practice to solve problems under uncertainty is to use certainty-equivalent heuristics. These heuristics solve a deterministic version of the problem in which the random elements are replaced by their average values. The same approach can also be used for our problem. For example, for the case of a possible price drop in a given future period that the buyer will also benefit owing to MFC clauses in the contract, one can set the discount amount to be equal to the difference between the current price and the expected price in that period. Note that to be eligible for this discount, the buyer still has to abide by the rules of obtaining MFC status. For the case of spot price uncertainty, one can use the expected spot price in each period as the price for that period in the deterministic formulation. For the case of index pricing, one can index the product prices to the expected price of the commodity or index.

We consider two versions of the certainty-equivalent heuristic. In the first version, certainty-equivalent deterministic problem is solved only once at the beginning of the planning horizon and the decisions are never changed. In the second version, the deterministic problem is re-solved at the beginning of each period after random events for that period are observed. We next explain how one can compute the solution and obtain the expected cost for each heuristic.

\subsection{Static certainty-equivalent (SCE) heuristic}

In this heuristic, the problem is solved only once at the beginning of the horizon (at node 0 ) and the solution is followed, regardless of the actual realizations of the random events throughout the planning horizon. In order to compute the solution for the certainty-equivalent heuristic, one can solve the 
following mathematical programme.

$$
\begin{aligned}
& \min \sum_{i \in \mathcal{I}} \sum_{j \in \mathcal{N}_{i}} \sum_{t \in \mathcal{T}} \mu_{i j t} x_{i j t}+\sum_{i \in \mathcal{I}} \sum_{t \in \mathcal{T}} \eta_{i t} I_{i t}-\sum_{r \in \mathcal{D}} \sum_{k \in \mathcal{K}_{r}} \bar{\pi}_{k}^{0} y_{k}-\sum_{r \in \mathcal{L}} \bar{\omega}_{r}^{0} z_{r} \\
& \text { s.t. } I_{i t}=I_{i, t-1}+\sum_{j \in \mathcal{N}_{i}} x_{i j t}-\delta_{i t} \quad \forall i \in \mathcal{I}, t \in \mathcal{T} \text {, } \\
& x_{i j t} \leqslant \kappa_{i j t} \quad \forall i \in \mathcal{I}, j \in \mathcal{N}_{i}, t \in \mathcal{T}, \\
& \sum_{i \in \mathcal{I}_{c}} \sum_{t \in \mathcal{T}_{c}} x_{i j(r) t} \geqslant \rho_{c} z_{r} \quad \forall r \in \mathcal{R}, c \in \mathcal{C}_{r}, \\
& y_{k} \leqslant \sum_{i \in \mathcal{I}_{k}} \sum_{t \in \mathcal{T}_{k}} x_{i j(r) t}-\theta_{k} z_{r} \quad \forall r \in \mathcal{D}, k \in \mathcal{K}_{r}, \\
& y_{k} \leqslant\left(\sum_{i \in \mathcal{I}_{k}} \sum_{t \in \mathcal{T}_{k}} \kappa_{i j(r) t}-\theta_{k}\right) z_{r} \quad \forall r \in \mathcal{D}, k \in \mathcal{K}_{r}, \\
& z_{r}+z_{r^{\prime}} \leqslant 1 \quad \forall\left\{r, r^{\prime}\right\} \in \mathcal{E}, \\
& x_{i j t} \geqslant 0 \quad \forall i \in \mathcal{I}, j \in \mathcal{N}_{i}, t \in \mathcal{T} \text {, } \\
& I_{i t} \geqslant 0 \quad \forall i \in \mathcal{I}, t \in \mathcal{T}, \\
& z_{r} \in\{0,1\} \quad \forall r \in \mathcal{R}, \\
& y_{k} \geqslant 0 \quad \forall r \in \mathcal{D}, k \in \mathcal{K}_{r} .
\end{aligned}
$$

Note that the formulation above is the same as the model in (1-11), except that the scenarios are removed. In this formulation, $x_{i j t}$ is the order quantity for item $i$ from supplier $j$ in period $t$ and $I_{i t}$ is the ending inventory for item $i$ in period $t$. The binary variable $z_{r}$ takes value 1 if rule $r \in \mathcal{R}$ applies and takes value 0 otherwise. The variable $y_{k}$ is the total amount of items in the set $\mathcal{I}_{k}$ that are discounted with discount $k \in \mathcal{K}_{r}$ as a part of rule $r \in \mathcal{D}$. The only parameters that are different from the model in (1-11) are $\bar{\pi}_{k}^{0}$, which stands for the expected value of the discount given by the discount $k$ and $\bar{\omega}_{r}^{0}$, which stands for the expected lump sum discount given by the rule $r$. Both of these expectations are taken at node 0 (unconditional expectation).

Let $x_{i j t}^{0}$ be the optimal order quantity for item $i$ from supplier $j$ in period $t$ obtained by solving (12-22) (superscript 0 is used to denote that this is the certainty-equivalent solution obtained at node 0$)$. Note that the optimal value of the model in (12-22) is not the true expected value obtained by following the ordering decisions obtained by solving (12-22). To calculate the expected cost of the certainty-equivalent heuristic, we need to reinstate the scenarios and solve the following mathematical programme.

$$
\begin{gathered}
\min \sum_{s \in \mathcal{V}} \gamma_{s}\left(\sum_{i \in \mathcal{I}} \sum_{j \in \mathcal{N}_{i}} \mu_{i j \tau(s)} x_{i j s}+\sum_{i \in \mathcal{I}} \eta_{i \tau(s)} I_{i s}-\sum_{r \in \mathcal{D}^{s}} \sum_{k \in \mathcal{K}_{r}} \pi_{k} y_{k}^{s}-\sum_{r \in \mathcal{L}^{s}} \omega_{r} z_{r}^{s}\right) \\
\text { s.t. }(2-11), \\
x_{i j s}=x_{i j \tau(s)}^{0} \quad \forall s \in \mathcal{V}, i \in \mathcal{I}, j \in \mathcal{N}_{i} .
\end{gathered}
$$

\subsection{Dynamic certainty-equivalent (DCE) heuristic}

In this heuristic, the problem is re-solved at the beginning of each period to account for realizations of all random events and prior decisions until that node. At node 0 , we solve the same model given in (12-22). We then need to re-solve the problem at every node (going layer by layer) as well. At node $s$, we need to solve the following mathematical programme.

$$
\begin{array}{r}
\min \sum_{i \in \mathcal{I}} \sum_{j \in \mathcal{N}_{i}} \sum_{t \in \mathcal{T}} \mu_{i j} x_{i j t}+\sum_{i \in \mathcal{I}} \sum_{t \in \mathcal{T}} \eta_{i t} I_{i t} \\
-\sum_{r \in \mathcal{D}} \sum_{k \in \mathcal{K}_{r}} \bar{\pi}_{k}^{s} y_{k}-\sum_{r \in \mathcal{L}} \bar{\omega}_{r}^{s} z_{r} \\
\text { s.t. }(13-22), \\
x_{i j \tau(\hat{s})}=x_{i j \hat{s}}^{\hat{s}} \quad \forall \hat{s} \in \mathcal{P}^{s}, i \in \mathcal{I}, j \in \mathcal{N}_{i} .
\end{array}
$$

The last set of constraints enforces that the order quantity decisions taken before node $s$ (nodes in the path from node 0 to node $s, \mathcal{P}^{s}$ ) are followed. Note also that we use $\bar{\pi}_{k}^{s}$ for the expected discount given by discount $k$ and $\bar{\omega}_{r}^{s}$ for the expected lump sum amount given by rule $r$. These reflect the fact that expectations are taken at node $s$ (conditioning on the fact that we are already at node $s$ ). If a discount or a rule depends only on random events that materialize in time periods before and at node $s(t \leqslant \tau(s))$, this means that we know these parameters with certainty at node $s$.

Let $x_{i j t}^{s}$ be the optimal order quantity for item $i$ from supplier $j$ in period $t$ obtained by solving model $(23,13-22,24)$ at node $s$. In order to compute the expected cost of the DCE heuristic, we need to solve the following programme.

$$
\begin{gathered}
\min \sum_{s \in \mathcal{V}} \gamma_{s}\left(\sum_{i \in \mathcal{I}} \sum_{j \in \mathcal{N}_{i}} \mu_{i j \tau(s)} x_{i j s}+\sum_{i \in \mathcal{I}} \eta_{i \tau(s)} I_{i s}-\sum_{r \in \mathcal{D}^{s}} \sum_{k \in \mathcal{K}_{r}} \pi_{k} y_{k}^{s}-\sum_{r \in \mathcal{L}^{s}} \omega_{r} z_{r}^{s}\right) \\
\text { s.t. }(2-11), \\
x_{i j s}=x_{i j \tau(s)}^{s} \quad \forall s \in \mathcal{V}, i \in \mathcal{I}, j \in \mathcal{N}_{i} .
\end{gathered}
$$

The last constraint enforces that order quantity decisions at each node are taken according to the solution of the certaintyequivalent model at that node.

\section{Application}

We apply our model in a preliminary study at a major manufacturing company. The company needs to procure various components that are used in the assembly of a large number of end-products in various manufacturing sites across the globe. Sourcing decisions are usually taken quarterly by holding bidding events. We test our model and the implications of its use in three bidding events that took place in 2010. These events are held for 40-45 items (components) and involve 3-5 suppliers. For most of the items, there are two or 
more suppliers that are competing for the buyer's business. Each supplier provides a base price for each offered item. In addition, suppliers also offer discounts that reflect their economies of scale in costs and market share targets. Each discount offer requires a minimum volume or a minimum spend on an item or a group of items and provides a discount on a set of items (which can be different from the set that the conditions are imposed on). The discounts are either incremental or all-units discounts.

In addition to these usual discount offers, at least one supplier in each bidding event provides most favoured customer benefits in its offers. If the supplier offering the MFC clause reduces the price in the middle of the quarter to other customers, it would extend the price reduction to the buyer in consideration as well if the buyer has already procured a minimum fraction of its demand (for this item or a group of items) from the supplier until that time. In order to model these possible discounts, we split the quarter into two periods and created scenarios to represent possible price reductions in items for which MFC clauses apply.

All MILPs in this section were implemented and solved using Gurobi (2012) version 5.0 on a notebook computer that has an Intel Dual-Core i7 6.20 GHz processor, 4 GB memory and a Windows 7 operating system. For all problems in our case study, the solution times were less than 2 seconds.

Table 1 summarizes our tests for these three bidding events. The buyer gets price and discount offers from the suppliers in two rounds. The suppliers submit initial bids in round 1 and revise them after getting some feedback from the buyer's procurement organization. We represent each round separately in Table 1 ( $i$ a and $i \mathrm{~b}$ stand for the first and second round offers for bidding event $i$, respectively). Our baseline for each event disregards all discounts offers (regular and MFC clauses). This corresponds to selecting the supplier that offers the minimum base price for each item.

In our first test for each bidding event, we run a version of our model where we ignore all MFC clauses. In the last three tests, we run our model considering the MFC clauses in the contracts. For each event, we consider three sub-scenarios: the prices for the items covered in MFC clauses may drop with a low probability, a medium probability and a high probability. The entries in Table 1 show the reduction in total procurement costs (as a percentage of total procurement costs when all discount offers are ignored). First, notice that regular discount offers lead to savings in the range of $0.93-3.23 \%$ for the company. In absolute terms, these savings are substantial for the company.

Optimizing sourcing decisions by also considering MFC clauses leads to important additional savings for bidding events 1 and 3. Even under a scenario when the price drops are not very likely, MFC clauses lead to additional savings up to $0.48 \%$ of the total spend. When the price drop probability is medium, the additional savings for bidding events 1 and 3 are in the range of $0.55-1.20 \%$. When the price drops are considered very likely, the incremental savings are in the range 1.05-1.92\%. Under some scenarios, incremental savings through MFC clauses are more than savings that are possible with only regular discount offers. Once again, these additional percentage gains correspond to substantial monetary savings for the company. In event 2 , the base prices and regular discount offers given by the MFC suppliers are either already very competitive (leading the buyer to select them even without MFC clauses) or very uncompetitive (leading the buyer to select other suppliers despite the possible MFC benefits).

\subsection{Detailed analysis}

In order to test our model and the performance of heuristics proposed in Section 5 in various other settings, we study bidding event $1 \mathrm{a}$ and variations of it in more detail. This event was held for 44 items. There were four qualified suppliers, named in the following as $A, B, C$ and $D$. Table 2 shows the number of items offered by different groups of suppliers. For example, four items are offered by all suppliers, while three items are offered by suppliers $A, B$ and $C$ but not $D$. For 29 items, 2 or more suppliers compete for the buyer's spend. There may be considerable differences between the prices offered by competing suppliers. In this event, the maximum bid can be as much as $34.71 \%$ higher than the minimum bid. On average, the maximum bids are $8.29 \%$ higher than the minimum bids. Note that there are 15 items that are offered by a single supplier (13 by supplier $A$ and 2 by supplier $B$ ), but they still cannot be removed from the model and solved independently since some of the discount offers and MFC conditions involve these items together with other items offered by multiple firms.

Table 1 The impact of discount offers and MFC clauses on total spend

\begin{tabular}{|c|c|c|c|c|c|c|}
\hline \multirow[t]{2}{*}{ Test } & \multicolumn{6}{|c|}{ Events } \\
\hline & $1 a$ & $1 b$ & $2 a$ & $2 b$ & $3 a$ & $3 b$ \\
\hline Ignore MFC clauses (regular discount offers only) & 0.93 & 1.26 & 2.29 & 2.31 & 3.22 & 3.23 \\
\hline \multicolumn{7}{|l|}{$\begin{array}{l}\text { Consider MFC clauses when the probability } \\
\text { of a price drop is }\end{array}$} \\
\hline Low & 0.97 & 1.67 & 2.29 & 2.31 & 3.70 & 3.71 \\
\hline Medium & 1.48 & 2.29 & 2.29 & 2.31 & 4.42 & 4.42 \\
\hline High & 1.98 & 2.91 & 2.30 & 2.32 & 5.14 & 5.13 \\
\hline
\end{tabular}


Table 2 Suppliers and their offerings in bidding event 1

\begin{tabular}{lccccccc}
\hline Suppliers & $A, B, C, D$ & $A, B, C$ & $A, B$ & $A, C$ & $A, D$ & $A$ & $C$ \\
\hline Number of items & 4 & 3 & 11 & 4 & 7 & 13 & 2 \\
\hline
\end{tabular}

Apart from base prices, suppliers propose various discount offers. In particular, supplier $A$ submits four, supplier $B$ submits three and supplier $C$ submits one discount offers. The offers involve multiple conditions on how much the buyer should buy from a set of items to qualify for the discount. The number of items in the condition sets is between 6 and 21. The discounts usually apply to the same set of items. However, some offers may apply a discount on items that are not in the condition set. Each offer provides a price reduction between $3 \%$ and $7 \%$ of the base price.

In addition to these eight discount offers, supplier $A$ is offering an MFC term for four items in its contract. According to this term, if the buyer procures a certain percentage of total demand from supplier $A$, supplier $A$ will ensure that the buyer will get the lowest price throughout the quarter. That is, if supplier $A$ drops the price for some or all of the items during the quarter, the buyer will also benefit from these price drops. We consider various scenarios for the drop in prices for these four items.

Since the total procurement spends are usually very large, we measure the effect of MFC clauses on procurement costs as a percentage of the savings obtained through regular discount offers. Let $S$ be the total procurement spend in the absence of any discount offers. Let $D$ be the procurement spend when only regular discount offers are utilized (those offers that are granted, regardless of the price drops to other customers). In this particular event, $S-D$ is about $0.9321 \%$ of $S$. That is, regular discount offers lead to $0.9321 \%$ cost savings. Let $D^{*}$ be the optimal expected procurement spend when MFC clauses are also used. Then we denote the effect of MFC terms as

$$
J^{*}=100 \times \frac{D-D^{*}}{S-D} .
$$

That is, $J^{*}$ measures the additional benefit of considering MFC clauses as a percentage of savings through regular discount offers.

Let $D_{S C E}$ and $D_{D C E}$ be the expected procurement spend if SCE and DCE heuristics are used, respectively. Then, we measure the regrets of these heuristics (given that MFC terms provide savings, ie, $D^{*}<D$ ) as follows

$$
\Delta_{S C E}=100 \times \frac{D_{S C E}-D^{*}}{D-D^{*}} \quad \Delta_{D C E}=100 \times \frac{D_{D C E}-D^{*}}{D-D^{*}} .
$$

We first consider two-period instances with different scenario trees. In our simplest experiment, two scenarios are considered: the prices drop by $\pi$ units in Period 2 with probability $\gamma$ or the

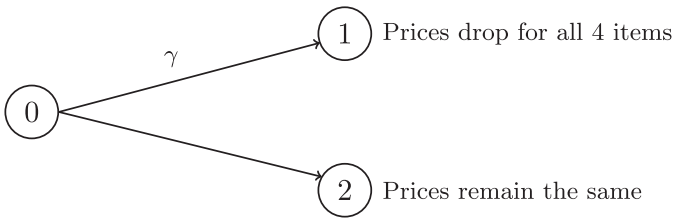

Figure 2 Scenario tree for Experiment 1.

\begin{tabular}{|c|c|c|c|c|c|c|}
\hline \multirow[b]{2}{*}{$\gamma$} & \multicolumn{3}{|c|}{ Discount amount $5 \%$} & \multicolumn{3}{|c|}{ Discount amount $10 \%$} \\
\hline & $J^{*}$ & $\Delta_{S C E}$ & $\Delta_{D C E}$ & $J^{*}$ & $\Delta_{S C E}$ & $\Delta_{D C E}$ \\
\hline 0.1 & 0.00 & 0.00 & 0.00 & 0.00 & 0.00 & 0.00 \\
\hline 0.2 & 0.00 & 0.00 & 0.00 & 3.81 & 100.00 & 100.00 \\
\hline 0.3 & 0.00 & 0.00 & 0.00 & 21.99 & 84.12 & 0.00 \\
\hline 0.4 & 0.00 & 0.00 & 0.00 & 40.17 & 46.79 & 0.00 \\
\hline 0.5 & 3.36 & 100.00 & 100.00 & 58.35 & 32.72 & 0.00 \\
\hline 0.6 & 10.54 & 66.84 & 0.00 & 76.53 & 23.35 & 0.00 \\
\hline 0.7 & 17.72 & 29.82 & 0.00 & 94.71 & 14.15 & 0.00 \\
\hline 0.8 & 24.90 & 14.15 & 0.00 & 112.89 & 7.91 & 0.00 \\
\hline 0.9 & 32.08 & 5.49 & 0.00 & 131.06 & 3.41 & 0.00 \\
\hline Avg & 17.72 & 43.26 & 20.00 & 67.44 & 39.06 & 12.50 \\
\hline
\end{tabular}

Table 3 Results for Experiment 1

prices remain the same with probability $1-\gamma$. The corresponding scenario tree is depicted in Figure 2.

The results are given in Table 3 for different values of $\gamma$ and $\pi$. Averages are reported in the last row of the table where the averages are taken over instances for which $J^{*}>0$. First, notice that MFC terms lead to important savings in this bidding event and, as expected, the benefits increase as the probability or the magnitude of price drops increase. The condition of the MFC clause is on the amount ordered in the first period. Consequently, the SCE and DCE heuristics behave the same way in the first period. The dynamic heuristic has the possibility to correct its decision by recourse in the second period. When $\gamma$ is small, neither the stochastic optimization model nor the heuristics choose to fulfill the condition of the MFC clause, leading to zero optimality gap for the heuristics. However, when $\gamma$ is large enough so that it is optimal to satisfy the condition of the MFC clause, then the gain obtained using the stochastic optimization model is greater than that of the SCE heuristic in all cases. The DCE heuristic obtains the same amount of reduction as our stochastic programming model if it opts for satisfying the MFC condition in the first period. Hence, there is a difference between the cost reductions obtained with these two methods only when the stochastic model chooses to satisfy the MFC condition and the dynamic heuristic does not (when $\gamma=0.5$ for a $5 \%$ decrease and when $\gamma=0.2$ for a $10 \%$ decrease). In these cases, $\Delta_{D C E}=100 \%$. One final observation is that the performance of the SCE heuristic is always worse than or equal to that of the DCE heuristic.

We can also use our model to fully characterize the regions in which the buyer should opt for the supplier's MFC clause and procure enough to satisfy the condition for MFC status. This 


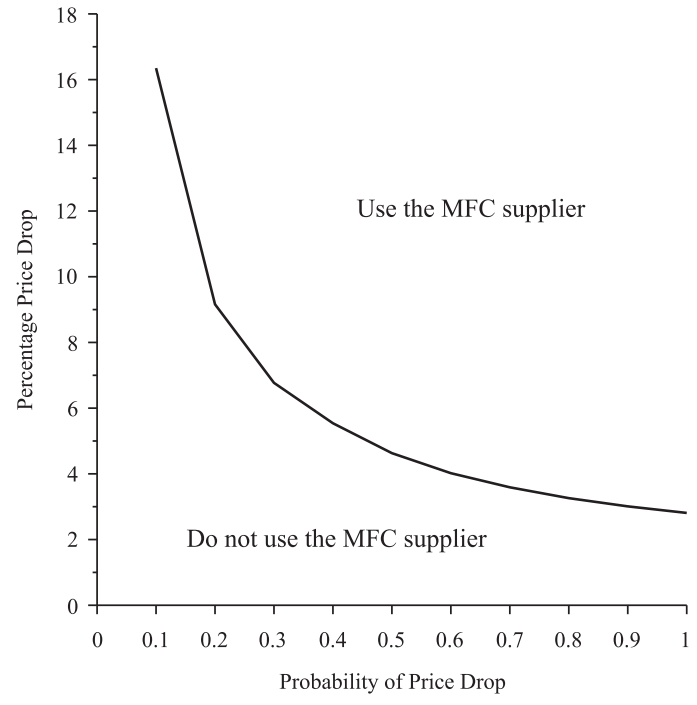

Figure 3 Indifference curve for selecting the MFC supplierExperiment 1.

can be accomplished by solving the model repeatedly to generate indifference curves. Figure 3 shows the indifference curve for Experiment 1. For every value of $\gamma$, we find the percentage price drop at which buying enough from the MFC supplier to qualify for a discount would lead to expected cost that is equal to what the buyer would get by only using regular discount offers. This curve can be used to guide the buyer's decision, given the estimates for the probability and magnitude of a price drop. On the indifference curve (eg, when the probability of price drop is 0.4 and magnitude of price drop is $5.54 \%$ ), the buyer is indifferent between satisfying the MFC condition and not. If the probability and/or the magnitude of the price drop are higher, the buyer should satisfy the MFC condition.

We next run another two-period experiment in which the prices may drop in different amounts: $5 \%$ or $10 \%$ of the original price. The scenario tree for this experiment is given in Figure 4. The results for various values of $\gamma_{1}$ and $\gamma_{2}$ are provided in Table 4. Once again, the possibility of a MFC status provides important benefits. The performances of the SCE and DCE heuristics are similar to those seen for Experiment 1. SCE heuristic may lead to significant suboptimality in a variety of settings especially when the price drop probability is small. DCE heuristic, on the other hand, captures either all or none of the additional savings possible with MFC terms.

In the third experiment, we group the items into two groups and assume that each group's price drops or stays the same independently from the other group. The scenario tree for this experiment is given in Figure 5.

The results for various values of $\gamma_{1}, \gamma_{2}$ and $\gamma_{3}$ are provided in Table 5. The results are not structurally different from the first two experiments, except that we now have more instances where the certainty-equivalent heuristic cannot capture any of the benefits of MFC terms.

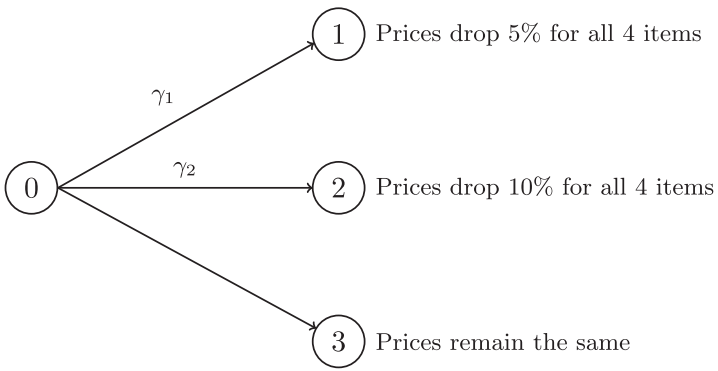

Figure 4 Scenario tree for Experiment 2.

Table 4 Results for Experiment 2

\begin{tabular}{rrrrrrccrc}
\hline$\gamma_{1}$ & $\gamma_{2}$ & $J^{*}$ & $\Delta_{S C E}$ & $\Delta_{D C E}$ & $\gamma_{1}$ & $\gamma_{2}$ & $J^{*}$ & $\Delta_{S C E}$ & $\Delta_{D C E}$ \\
\hline 0.1 & 0.1 & 0.00 & 0.00 & 0.00 & 0.3 & 0.3 & 43.53 & 30.36 & 0.00 \\
0.2 & 0.1 & 0.00 & 0.00 & 0.00 & 0.4 & 0.3 & 50.71 & 22.59 & 0.00 \\
0.3 & 0.1 & 7.18 & 100.00 & 100.00 & 0.5 & 0.3 & 57.89 & 16.75 & 0.00 \\
0.4 & 0.1 & 14.36 & 75.66 & 0.00 & 0.1 & 0.4 & 47.35 & 35.98 & 0.00 \\
0.5 & 0.1 & 21.54 & 42.26 & 0.00 & 0.2 & 0.4 & 54.53 & 28.01 & 0.00 \\
0.1 & 0.2 & 10.99 & 100.00 & 100.00 & 0.3 & 0.4 & 61.71 & 21.90 & 0.00 \\
0.2 & 0.2 & 18.17 & 80.78 & 0.00 & 0.4 & 0.4 & 68.89 & 14.85 & 0.00 \\
0.3 & 0.2 & 25.35 & 50.96 & 0.00 & 0.5 & 0.4 & 76.07 & 8.00 & 0.00 \\
0.4 & 0.2 & 32.53 & 34.30 & 0.00 & 0.1 & 0.5 & 65.53 & 26.45 & 0.00 \\
0.5 & 0.2 & 39.71 & 23.67 & 0.00 & 0.2 & 0.5 & 72.71 & 19.32 & 0.00 \\
0.1 & 0.3 & 29.17 & 57.38 & 0.00 & 0.3 & 0.5 & 79.89 & 12.40 & 0.00 \\
0.2 & 0.3 & 36.35 & 41.20 & 0.00 & 0.4 & 0.5 & 87.07 & 6.62 & 0.00 \\
& & & & & & Avg & 45.51 & 38.61 & 9.09 \\
\hline
\end{tabular}

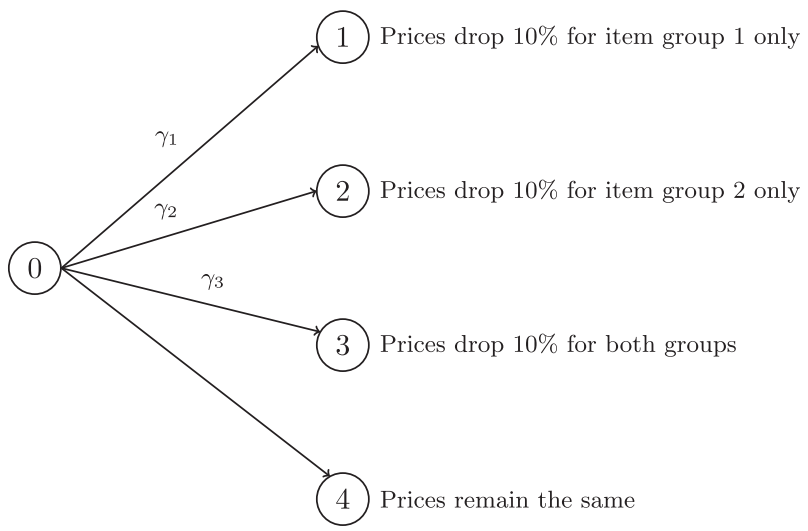

Figure 5 Scenario tree for Experiment 3.

Our final experiment models three periods. Reflecting the regular pattern in practice, the demand in each of the periods 1 and 2 is assumed to be $30 \%$ of the total demand, while demand in Period 3 is assumed to be $40 \%$. Figure 6 shows the scenario tree for this experiment. In order to obtain the MFC status and benefit from a possible price drop in Period 2, a minimum amount should be purchased in Period 1. In order to obtain the MFC status and benefit from a possible price drop in period 3, the sum of purchases in periods 2 and 3 should be above another threshold. 
Table 5 Results for Experiment 3

\begin{tabular}{|c|c|c|c|c|c|c|c|c|c|c|c|}
\hline$\gamma_{1}$ & $\gamma_{2}$ & $\gamma_{3}$ & $J^{*}$ & $\Delta_{S C E}$ & $\Delta_{D C E}$ & $\gamma_{1}$ & $\gamma_{2}$ & $\gamma_{3}$ & $J^{*}$ & $\Delta_{S C E}$ & $\Delta_{D C E}$ \\
\hline 0.1 & 0.1 & 0.0 & 0.00 & 0.00 & 0.00 & 0.1 & 0.2 & 0.1 & 7.99 & 100.00 & 100.00 \\
\hline 0.2 & 0.1 & 0.0 & 0.00 & 0.00 & 0.00 & 0.1 & 0.3 & 0.1 & 12.16 & 100.00 & 100.00 \\
\hline 0.3 & 0.1 & 0.0 & 13.65 & 98.98 & 0.00 & 0.1 & 0.4 & 0.1 & 16.33 & 100.00 & 100.00 \\
\hline 0.4 & 0.1 & 0.0 & 27.66 & 44.03 & 0.00 & 0.2 & 0.2 & 0.1 & 21.99 & 84.12 & 0.00 \\
\hline 0.1 & 0.2 & 0.0 & 0.00 & 0.00 & 0.00 & 0.2 & 0.3 & 0.1 & 26.17 & 76.94 & 0.00 \\
\hline 0.2 & 0.2 & 0.0 & 3.81 & 100.00 & 100.00 & 0.3 & 0.3 & 0.1 & 40.17 & 46.79 & 0.00 \\
\hline 0.3 & 0.2 & 0.0 & 17.82 & 94.65 & 0.00 & 0.4 & 0.4 & 0.1 & 58.35 & 32.72 & 0.00 \\
\hline 0.4 & 0.2 & 0.0 & 31.83 & 48.81 & 0.00 & 0.0 & 0.0 & 0.2 & 3.81 & 100.00 & 100.00 \\
\hline 0.1 & 0.3 & 0.0 & 0.00 & 0.00 & 0.00 & 0.1 & 0.1 & 0.2 & 21.99 & 84.12 & 0.00 \\
\hline 0.2 & 0.3 & 0.0 & 7.99 & 100.00 & 100.00 & 0.2 & 0.2 & 0.2 & 40.17 & 46.79 & 0.00 \\
\hline 0.3 & 0.3 & 0.0 & 21.99 & 84.12 & 0.00 & 0.3 & 0.3 & 0.2 & 58.35 & 32.72 & 0.00 \\
\hline 0.4 & 0.3 & 0.0 & 36.00 & 47.68 & 0.00 & 0.0 & 0.0 & 0.3 & 21.99 & 84.12 & 0.00 \\
\hline 0.1 & 0.4 & 0.0 & 0.00 & 0.00 & 0.00 & 0.1 & 0.1 & 0.3 & 40.17 & 46.79 & 0.00 \\
\hline 0.2 & 0.4 & 0.0 & 12.16 & 100.00 & 100.00 & 0.2 & 0.2 & 0.3 & 58.35 & 32.72 & 0.00 \\
\hline 0.3 & 0.4 & 0.0 & 26.17 & 76.94 & 0.00 & 0.3 & 0.3 & 0.3 & 76.53 & 23.35 & 0.00 \\
\hline 0.4 & 0.4 & 0.0 & 40.17 & 46.79 & 0.00 & 0.0 & 0.0 & 0.4 & 40.17 & 46.79 & 0.00 \\
\hline 0.1 & 0.1 & 0.1 & 3.81 & 100.00 & 100.00 & 0.1 & 0.1 & 0.4 & 58.35 & 32.72 & 0.00 \\
\hline 0.2 & 0.1 & 0.1 & 17.82 & 94.65 & 0.00 & 0.2 & 0.2 & 0.4 & 76.53 & 23.35 & 0.00 \\
\hline 0.3 & 0.1 & 0.1 & 31.83 & 48.81 & 0.00 & 0.0 & 0.0 & 0.5 & 58.35 & 32.72 & 0.00 \\
\hline \multirow[t]{2}{*}{0.4} & 0.1 & 0.1 & 45.83 & 30.98 & 0.00 & 0.1 & 0.1 & 0.5 & 76.53 & 23.35 & 0.00 \\
\hline & & & & & & & & Avg & 32.94 & 64.76 & 22.22 \\
\hline
\end{tabular}

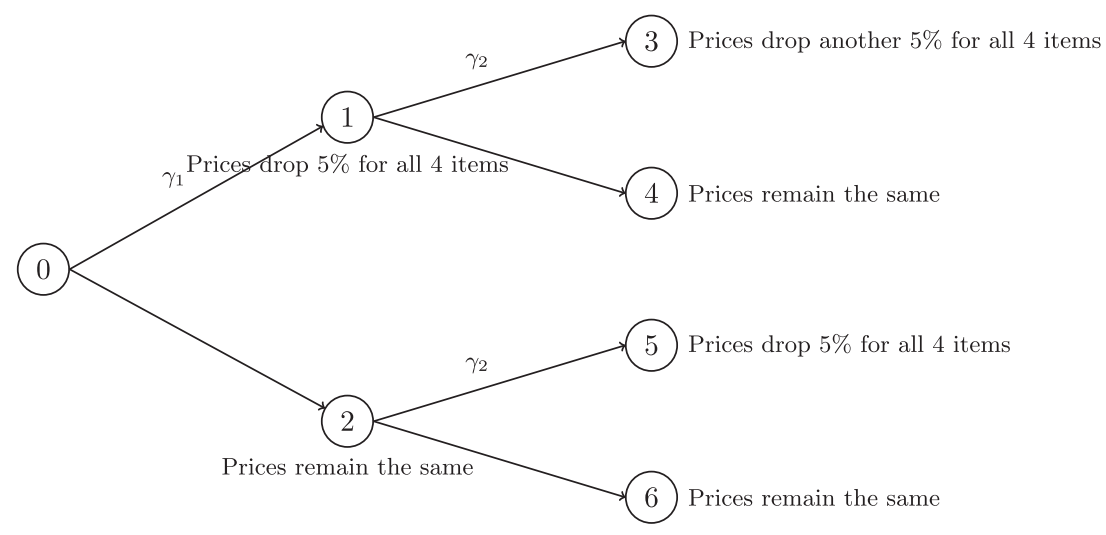

Figure 6 Scenario tree for Experiment 4.

The results of Experiment 4 are shown in Table 6 for different values of $\gamma_{1}$ and $\gamma_{2}$. Considering MFC clauses still leads to important savings in the three-period model and savings increase as the probability of a price drop increases. The percentage gap of the SCE heuristic can be significant, especially when the price drop probability is low. Structurally, the performance of the DCE heuristic in the three-period problem is different than what we observe in the two-period problem. It is now possible that DCE heuristic lead to a gap other than 0 and $100 \%$.

In Table 7, we summarize the results of our four experiments. For each experiment, the column labeled \# states the number of valid instances (ie, those for which MFC terms lead to additional savings). For each heuristic, the column labeled $\operatorname{Avg}(\Delta)$ shows the average regret, the column labeled $\mathrm{F}$ shows
Table 6 Results for Experiment 4

\begin{tabular}{lcrrr}
\hline$\gamma_{1}$ & $\gamma_{2}$ & $J^{*}$ & $\Delta_{S C E}$ & $\Delta_{D C E}$ \\
\hline 0.05 & 0.05 & 0.00 & 0.00 & 0.00 \\
0.10 & 0.10 & 1.87 & 100.00 & 100.00 \\
0.15 & 0.15 & 6.71 & 85.92 & 2.50 \\
0.20 & 0.20 & 11.17 & 53.93 & 1.76 \\
0.25 & 0.25 & 15.28 & 36.28 & 1.40 \\
0.30 & 0.30 & 19.09 & 27.13 & 1.17 \\
0.35 & 0.35 & 22.63 & 21.42 & 1.00 \\
0.40 & 0.40 & 25.93 & 17.14 & 0.87 \\
0.45 & 0.45 & 29.00 & 14.26 & 0.75 \\
0.50 & 0.50 & 31.88 & 12.28 & 0.64 \\
0.55 & 0.55 & 34.57 & 10.90 & 0.54 \\
0.60 & 0.60 & 37.09 & 8.55 & 0.45 \\
& Avg & 21.38 & 32.26 & 10.10 \\
\hline
\end{tabular}


Table 7 Summary of experiments

\begin{tabular}{|c|c|c|c|c|c|c|c|c|}
\hline \multirow[b]{2}{*}{ Experiments } & \multirow[b]{2}{*}{ Description } & \multirow[b]{2}{*}{$\#$} & \multicolumn{3}{|c|}{$S C E$} & \multicolumn{3}{|c|}{$D C E$} \\
\hline & & & $\operatorname{Avg}(\Delta)$ & $F$ & $S$ & $\operatorname{Avg}(\Delta)$ & $F$ & $S$ \\
\hline 1a & Two periods: prices for four items drop by $5 \%$ & 5 & 43.26 & 1 & 0 & 20.00 & 1 & 4 \\
\hline $1 b$ & Two periods: prices for four items drop by $10 \%$ & 8 & 39.06 & 1 & 0 & 12.50 & 1 & 7 \\
\hline 2 & Two periods: prices for four items drop by $5 \%$ or $10 \%$ & 22 & 38.61 & 2 & 0 & 9.09 & 2 & 20 \\
\hline 3 & Two periods: prices for two or four items drop by $10 \%$ & 35 & 64.76 & 8 & 0 & 22.22 & 8 & 27 \\
\hline 4 & Three periods: prices for four items drop by $5 \%$ first, then another $5 \%$ & 11 & 32.26 & 1 & 0 & 10.10 & 1 & 0 \\
\hline All & & 81 & 49.38 & 13 & 0 & 15.91 & 13 & 58 \\
\hline
\end{tabular}

the number of instances where the regret is $100 \%$ (ie, the heuristic does not generate any savings from MFC terms) and the column $\mathrm{S}$ shows the number of instances where the regret is $0 \%$ (ie, the heuristic finds an optimal solution). On average, SCE heuristic leads to a regret of $49.38 \%$ and DCE heuristic leads to a regret of $15.91 \%$. Out of all 81 instances, SCE heuristic leads to 0 savings in 13 instances. SCE heuristic cannot find an optimal solution in any of the 81 instances. DCE heuristic leads to 0 savings in 13 instances as well. In 58 instances, DCE heuristic finds an optimal solution.

\section{Conclusion}

In this paper, we study the problem of a buyer who has to procure large volumes of multiple items over multiple periods and needs to evaluate discount offers from multiple suppliers for this purpose. Some of these discounts are tied to future realizations of random events. The objective of the buyer is to minimize his expected procurement and inventory holding costs subject to satisfying its demand and other various side constraints. We formulate the problem as a scenario-based multi-stage stochastic optimization model. The formulation is very general in the sense that we can model various random events such as a supplier dropping price for other customers or a change in a price-index or spot price of a commodity. We can also model very complex offers that are frequently observed in industry such as those that involve conditions on multiple items and periods and apply incremental or all-units discounts to multiple items and periods that are different from those for which the conditions are imposed on. The model also allows the buyer to carry inventory to benefit from a discount offer. We also propose two certaintyequivalent heuristics that can be used for this problem and show how we can evaluate the performance of these heuristics. We use our model in a preliminary study to see the effect of MFC status benefits on three bidding events that were administered by a global manufacturing company in 2010. The results show that taking the MFC terms into account using our model leads to significant savings for the company and using heuristics may fail to capture most of these savings.

There are many avenues for future research. First, one may consider modelling uncertainty on other parameters of the problem. Relevant uncertain parameters for procurement bidding events include item demands and supplier capacities. For some of these uncertain parameters, scenario-based formulations may not be adequate and other approaches may be necessary to model uncertainty. Second, one may relax the assumption that the buyer is risk-neutral and consider alternative risk profiles.

In our specific application, the MILPs were solved very quickly using an off-the-shelf solver. For larger problems, one may focus on developing efficient approaches. It may be possible to formulate a stronger model using particular structures of the discounts, as briefly discussed in Section 4. One can also consider using decomposition methods as is often done in solving large scale stochastic optimization problems.

\section{References}

Aissaoui N, Haouari M and Hassini E (2007). Supplier selection and order lot sizing modeling: A review. Computers and Operations Research 34(12): 3516-3540.

Anupindi R and Bassok Y (1998). Approximations for multiproduct contracts with stochastic demands and business volume discounts: Single supplier case. IIE Transactions 30(8): 723-734.

Avery S (2008). HP looks beyond price discounts. Purchasing Magazine 137(9): 55.

Bassok Y and Anupindi R (1997). Analysis of supply contracts with total minimum commitment. IIE Transactions 29(5): 373-381.

Benton W and Park S (1996). A classification of literature on determining the lot size under quantity discounts. European Journal of Operational Research 92(2): 219-238.

Bichler M, Schneider S, Guler K and Sayal M (2011). Compact bidding languages and supplier selection for markets with economies of scale and scope. European Journal of Operational Research 214(1): 67-77.

Carbone J (2004). Hewlett-Packard wins for the 2nd time. Purchasing Magazine 133(14): 34-50.

Chauhan SS, Eremeev AV, Romanova AA, Servakh VV and Woeginger GJ (2005). Approximation of the supply scheduling problem. Operations Research Letters 33(3): 249-254.

Chopra S and Meindl P (2013). Supply Chain Management: Strategy, Planning, and Operation. Pearson Education: Harlow, UK, 5th edn.

Crama Y, Pascual JR and Torres A (2004). Optimal procurement decisions in the presence of total quantity discounts and alternative product recipes. European Journal of Operational Research 159(1): 364-378.

DoD-ESI (2012). Blanket Purchase Agreement, DoD Enterprise Software Agreement (ESA), US Department of Defense, Enterprise Software Initiative. 
Goossens DR, Maas AJT, Spieksma FCR and van de Klundert JJ (2007) Exact algorithms for procurement problems under a total quantity discount structure. European Journal of Operational Research 178(2): 603-626.

Gurobi Optimizer Version 5.0 (2012). Gurobi Optimization, Inc., Houston, TX. (software program).

Katz P, Sadrian A and Tendick P (1994). Telephone companies analyze price quotations with Bellcore's PDSS software. Interfaces 24(1): $50-63$.

Manerba D and Mansini R (2012). An exact algorithm for the capacitated total quantity discount problem. European Journal of Operational Research 222(2): 287-300.

Mansini R, Savelsbergh M and Tocchella B (2012). The supplier selection problem with quantity discounts and truckload shipping. Omega 40(4): 445-455.

Montalbano E (2011). Oracle pays \$199.5 million to settle GSA claims. InformationWeek, 7 October.

Moody PE (2006). With supply management, technology rules! Supply Chain Management Review May-June: 41-48.

Munson CL and Rosenblatt MJ (1998). Theories and realities of quantity discounts: An exploratory study. Production and Operations Management 7(4): 352-369.

Murthy N, Soni S and Ghosh S (2004). A framework for facilitating sourcing and allocation decisions for make-to-order items. Decision Sciences 35(4): 609-637.

Nagali V et al (2008). Procurement risk management (PRM) at HewlettPackard company. Interfaces 38(1): 51-60.

Neumann A and von Hirschhausen C (2004). Less long-term gas to Europe? A quantitative analysis of European long-term gas supply. Zeitschrift für Energiewirtschaft 28(3): 175-182.

Qin H, Luo M, Gao X and Lim A (2012). The freight allocation problem with all-units quantity-based discount: A heuristic algorithm. Omega 40(4): 415-423.
Rong Y, Shen ZJ and Yano CA (2012). Cheaper by the pallet? Multiitem procurement with standard batch sizes. IIE Transactions $\mathbf{4 4}(6)$ : 405-418.

Sadrian A and Yoon Y (1994). A procurement decision support system in business volume discount environments. Operations Research 42(1): 14-23.

Sample Business Contracts (2012). Amendment to private label agreement and project development and license agreement between Cisco Systems, Inc. and Frontier Software Development, Inc.

Sandholm T (2007). Expressive commerce and its application to sourcing: How we conducted $\$ 35$ billion of generalized combinatorial auctions. AI Magazine 28(3): 45-58.

Sherr I (2011). Demand for PCs falters. Wall Street Journal 14 April.

Silver E, Pyke DF and Peterson R (1998). Inventory Management and Production Planning and Scheduling. 3rd edn. Wiley: New York.

Stadtler H (2007). A general quantity discount and supplier selection mixed integer programming model. OR Spectrum 29(4): 723-744.

Tempelmeier H (2002). A simple heuristic for dynamic order sizing and supplier selection with time-varying data. Production and Operations Management 11(4): 499-515.

van de Klundert J, Kuipers J, Spieksma F and Winkels M (2005). Selecting telecommunication carriers to obtain volume discounts. Interfaces 35(2): 124-132.

$\mathrm{Xu} \mathrm{J}, \mathrm{Lu} \mathrm{L}$ and Glover F (2000). The deterministic multi-item dynamic lot size problem with joint business volume discount. Annals of Operations Research 96(1-4): 317-337.

Received 26 March 2013; accepted 09 August 2013 after one revision 